\title{
Characteristic Roots of a Class of Fractional Oscillators
}

\author{
Ming Li, ${ }^{1,2}$ S. C. Lim, ${ }^{3}$ Carlo Cattani, ${ }^{4}$ and Massimo Scalia ${ }^{5}$ \\ ${ }^{1}$ School of Information Science \& Technology, East China Normal University No. 500, Dong-Chuan Road, Shanghai 200241, China \\ ${ }^{2}$ Department of Computer and Information Science, University of Macau, \\ Avenue Padre Tomas Pereira, Taipa 1356, Macau SAR, China \\ ${ }^{3}$ Faculty of Engineering, Multimedia University, Selangor Darul Ehsan, 63100 Cyberjaya, Malaysia \\ ${ }^{4}$ Department of Mathematics, University of Salerno, Via Ponte Don Melillo, 84084 Fisciano, Italy \\ ${ }^{5}$ Department of Mathematics, University of Rome, la Sapienza Piazzale Aldo Moro, 00185 Rome, Italy
}

Correspondence should be addressed to Ming Li; ming_lihk@yahoo.com

Received 9 August 2013; Accepted 13 September 2013

Academic Editor: Gongnan Xie

Copyright (C) 2013 Ming Li et al. This is an open access article distributed under the Creative Commons Attribution License, which permits unrestricted use, distribution, and reproduction in any medium, provided the original work is properly cited.

The fundamental theorem of algebra determines the number of characteristic roots of an ordinary differential equation of integer order. This may cease to be true for a differential equation of fractional order. The results given in this paper suggest that the number of the characteristic roots of a class of oscillators of fractional order may in general be infinitely great. Further, we infer that it may also be the case for the characteristic roots of a differential equation of fractional order greater than 1 . The relationship between the range of the fractional order and the locations of characteristic roots of oscillators in the complex plane is considered.

\section{Introduction}

Oscillators are an essential component in devices in electron positron collider systems (see, e.g., Zhao et al. [1], Ma et al. [2], Zang et al. [3], Ding et al. [4], Marder et al. [5], Barroso [6], Miller et al. [7], and Lemke [8], just citing a few). As a matter of fact, oscillations are phenomena widely observed in sciences and engineering relating to high energy physics (see, e.g., Akhmediev et al. [9], Bachas [10], Winter et al. [11], Dodonov [12], Tan [13], Diamandis et al. [14], Greenwald et al. [15], Mathews et al. [16], Faiman [17], Cocho et al. [18], Baldiotti et al. [19], Kyu Shin [20], Kirson [21], Clement [22], Sikström et al. [23], Asghari et al. [24], Um et al. [25], Bahar and Yasuk [26], Hassanabadi et al. [27], Bhattacharya and Roy [28], and Saad et al. [29], simply mentioning a few).

There are various structures of oscillators, such as Mathieu oscillator (Floris [30]), Liénard type oscillator (Yaşar [31]), relativistic oscillator (Osborne [32]), Schrödinger equation type oscillator (Cornwall and Tiktopoulos [33]), and Duffing oscillator (Baltanás et al. [34] and Erturk and Inman [35]). In fact, oscillators play a role in various fields, ranging from experimental physics to electronics engineering (see, e.g., Riley et al. [36], Soong and Grigoriu [37], Harris
[38], Papoulis [39], Bendat and Piersol [40], Devasahayam [41], Karrenberg [42], Edson [43], and Balaban et al. [44]).

This research is in the domain of fractional oscillators that attract increasing interests of physicists and engineers. More specifically, we aim at revealing specific properties of characteristic roots of a class of fractional oscillators. In doing so, we first consider an ordinary differential equation of order $n$ given by

$$
b_{n} \frac{d^{n} y(t)}{d t^{n}}+b_{n-1} \frac{d^{n-1} y(t)}{d t^{n-1}}+\cdots+b_{0}=x(t),
$$

where $n$ is a natural number and $b_{n}$ is any complex number. We always assume that at least one of the higher coefficients $b_{n} \neq 0$ for $n>1$. The characteristic equation of (1) is given by

$$
B(\alpha)=b_{n} \alpha^{n}+b_{n-1} \alpha^{n-1}+\cdots+b_{0}=0 .
$$

The fundamental theorem of algebra says that the number of roots of (2) is $n$ (G. A. Korn and T. M. Korn [45]). This theorem is stated in the domain of complex variables (Krantz [46]).

Suppose that the $n$ roots of $B(\alpha)$ are $\alpha_{1}, \alpha_{2}, \ldots, \alpha_{n}$. For each root $r$ of multiplicity of $m$, either real or complex, we 
always consider $r$ the $m$ roots in what follows unless otherwise stated. Using the partial fraction expansion, $B(\alpha)$ can be expressed by

$$
B(\alpha)=b_{n}\left(\alpha-\alpha_{1}\right)\left(\alpha-\alpha_{2}\right) \cdots\left(\alpha-\alpha_{n}\right)=b_{n} \prod_{i=1}^{n}\left(\alpha-\alpha_{i}\right) .
$$

Now, we rewrite (3) by the following expression:

$$
B(\alpha)= \begin{cases}K \prod_{j=1}^{n / 2}\left(m_{j} \alpha_{j}^{2}+c_{j} \alpha_{j}+k_{j}\right), & n \text { is even, } \\ K\left(\alpha-\alpha_{n}\right) \prod_{j=1}^{(n-1) / 2}\left(m_{j} \alpha_{j}^{2}+c_{j} \alpha_{j}+k_{j}\right), & n \text { is odd, }\end{cases}
$$

where $m_{j}, c_{j}, k_{j}$, and $K$ are constants. Without loss of generality, we can suppose that the only simple zero of $B(\alpha)$ is $\alpha_{n}$ if $n$ is odd.

The factor $\left(m_{j} \alpha_{j}^{2}+c_{j} \alpha_{j}+k_{j}\right)$ in (4) corresponds to the oscillator equation in the form

$$
m_{j} \frac{d^{2} y(t)}{d t^{2}}+c_{j} \frac{d y(t)}{d t}+k_{j} y(t)=x(t) .
$$

The characteristic equation of (5) is in the form

$$
\left(m_{j} \alpha_{j}^{2}+c_{j} \alpha_{j}+k_{j}\right)=0 .
$$

There are two classes of fractional oscillators. One is in the form (Ryabov and Puzenko [47, Eq. (1)], Ahmad et al. [48], Radwan et al. [49], Drozdov [50, Eq. (9)], Tofighi and Pour [51], Tofighi [52, Eq. (2)], Blaszczyk et al. [53, Eq. (10)], and Narahari Achar et al. [54, 55])

$$
\begin{gathered}
m_{j} \frac{d^{2 \pm \varepsilon} y(t)}{d t^{2 \pm \varepsilon}}+c_{j} \frac{d^{ \pm \varepsilon} y(t)}{d t^{ \pm \varepsilon}}+k_{j} y(t) \\
=x(t) \quad \text { for } 0 \leq \varepsilon<1 .
\end{gathered}
$$

The other is expressed with the form (Lim et al. [56-58], Muniandy and Lim [59], Eab and Lim [60], and Li et al. [61])

$$
\left(m_{j} D^{2} y(t)+c_{j} D y(t)+k_{j} y(t)\right)^{\beta}=x(t) \text { for } \beta>0 .
$$

This research utilizes the form of (8).

According to the fundamental theorem of algebra, there are only two characteristic roots with respect to the oscillator equation (5). They are

$$
\alpha_{j, 12}=\frac{-c_{j} \pm \sqrt{c_{j}^{2}-4 m_{j} k_{j}}}{2 m_{j}} .
$$

One might be carelessly misled to consider that there exist only two characteristic roots regarding the fractional oscillator equation (8) because

$$
\left(m_{j} \alpha_{j, 12}^{2}+c_{j} \alpha_{j, 12}+k_{j}\right)^{\beta}=0
$$

However, we shall show that the number of the roots in the above expression dramatically differs from what in the following expression:

$$
\left(m_{j} \alpha_{j, 12}^{2}+c_{j} \alpha_{j, 12}+k_{j}\right)=0
$$

The contributions of this paper are twofold. One is to exhibit that the number of the characteristic roots of (8) is in general infinitely great. The other is to reveal the relationship between the range of $\beta$ and the locations of the characteristic roots of (8) in a complex plane. In addition, if all $\alpha_{j}(j=$ $1, \ldots, n)$ are simple complex pair of roots, the ordinary differential equation of $\operatorname{order} n$ (1) and its generalization given by

$$
\begin{gathered}
\left(b_{n} D^{n} y(t)+b_{n-1} D^{n-1} y(t)+\cdots+b_{0}\right)^{\beta} \\
=x(t), \quad \beta>0
\end{gathered}
$$

may be taken as the product of oscillators of integer order and fractional order in series in the wide sense for $n$ being even, respectively.

The rest of the paper is organized as follows. We shall give the results in Section 2, including the proof that there are infinite characteristic roots regarding (8), and the explanation that (1) and (12) may be taken as oscillators in series in the wide sense. Discussions are given in Section 3 , which is followed by Conclusions.

\section{Results}

2.1. Result 1. The number of the characteristic roots of (8) may be infinitely great.

Denote by $\mathbf{C}$ the set of complex numbers. Let $z \in \mathbf{C}$. Suppose that a power function is given by

$$
w=z^{b}=e^{b \ln (z)}
$$

Then, the number of different values of $w$ relies on the value of $b$ for a given $z$. More precisely, we express that by the following lemmas, which can be found in the literature, such as [45] or $\mathrm{Yu}[62]$.

Lemma 1. If $b$ is a rational number expressed by the irreducible fraction $l / m$, where $m \geq 1$, the number of values of $z^{b}$ is $m$.

Lemma 2. If $b$ is an irrational number or imaginary number, the number of values of $z^{b}$ is infinitely great.

The general expression of $w$ is in the form

$$
\begin{aligned}
w & =z^{b}=e^{b \ln (z)} \\
& =e^{b[\ln |z|+i(\arg z+2 m \pi)]}, \quad i=\sqrt{-1} .
\end{aligned}
$$

Therefore, from Lemma 2, we have the theorem below.

Theorem 3. The number of the characteristic roots of the fractional oscillator (8) is infinitely great if $\beta \neq 1$. 
Proof. Let $c_{j}=0$. Then, the characteristic roots $\alpha_{j, 12}$ in (9) become the imaginary numbers expressed by

$$
\alpha_{j, 12}=\frac{ \pm i \sqrt{m_{j} k_{j}}}{m_{j}} .
$$

According to Lemma 2, the number of the roots of either $\left(\alpha-\alpha_{j, 1}\right)^{\beta}$ or $\left(\alpha-\alpha_{j, 2}\right)^{\beta}$ is infinitely great. Thus, Theorem 3 results.

2.2. Result 2. Equations (1) and (12) may be taken as oscillators in series.

Denote $\left(m_{j} \alpha_{j}^{2}+c_{j} \alpha_{j}+k_{j}\right)$ in (4) by $B_{j}(\alpha)$ :

$$
B_{j}(\alpha)=\left(m_{j} \alpha_{j}^{2}+c_{j} \alpha_{j}+k_{j}\right)
$$

Without loss of generality, $n$ is assumed to be even. In addition, we suppose that all $\alpha_{j}(j=1, \ldots, n)$ are simple complex pair of roots. Then, we have the theorem below.

Theorem 4. The ordinary differential equation (1) may be taken as an oscillator (i.e., product of oscillators) in the wide sense if $n$ is even and all $\alpha_{j}(j=1, \ldots, n)$ are simple complex pair of roots. By wide sense, one means that it is a system consisting of the product of a series of conventional 2-order oscillators.

Proof. On the one hand, $B_{j}(\alpha)$ stands for the characteristic equation of the $j$ th oscillator of order 2 since $n$ is even and all $\alpha_{j}(j=1, \ldots, n)$ are simple complex pair of roots. On the other hand, the characteristic equation of (1) can be expressed by

$$
B(\alpha)= \begin{cases}K \prod_{j=1}^{n / 2} B_{j}(\alpha), & n \text { is even, } \\ K\left(\alpha-\alpha_{n}\right) \prod_{j=1}^{(n-1) / 2} B_{j}(\alpha), & n \text { is odd. }\end{cases}
$$

Based on the theory of filter design (Mitra and Kaiser [63]), the system (1) in the case of $n$ being even may be expressed by Figure 1 .

Therefore, the system (1) may be expressed by the product of a series of 2-order oscillators.

Denote by $B^{\beta}(\alpha)$ the characteristic equation of (12). Then,

$$
B^{\beta}(\alpha)= \begin{cases}K \prod_{j=1}^{n / 2} B_{j}^{\beta}(\alpha), & n \text { is even, } \\ K\left(\alpha-\alpha_{n}\right) \prod_{j=1}^{(n-1) / 2} B_{j}^{\beta}(\alpha), & n \text { is odd, }\end{cases}
$$

where

$$
B_{j}^{\beta}(\alpha)=\left(m_{j} \alpha_{j}^{2}+c_{j} \alpha_{j}+k_{j}\right)^{\beta} .
$$

Thus, the system of fractional order expressed by (12) may be the product of a series of fractional oscillators of (8).

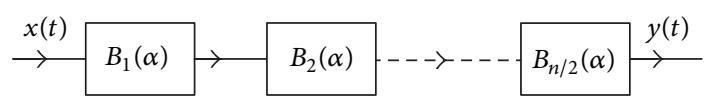

FIgURE 1: Oscillators of 2-order in concatenation.

\section{Discussions}

The previous section says that there are infinite roots in $B_{j}^{\beta}(\alpha)$ if $\beta \neq 1$. In the case of $\beta=1, B_{j}^{\beta}(\alpha)$ reduces to the characteristic equation of the conventional oscillator (5) with two roots only. Thus, the fraction $\beta \neq 1$ dramatically alters the behavior of characteristic roots of oscillators. For facilitating our discussions, we omit the subscript $j$ in what follows if not confused. More precisely, we specifically consider the fractional oscillator in the form

$$
\begin{gathered}
\left(m D^{2} y(t)+c D y(t)+k y(t)\right)^{\beta} \\
=x(t) \quad \text { for } \beta>0 .
\end{gathered}
$$

Figure 2 shows an RLC resonance circuit in series, where $R, L$, and $C$ represent resistor, inductor, and capacity, respectively. In Figure 2, $I(t)$ is the electronic current and $v(t)$ the power source. According to the Kirchhoff voltage law, one has

$$
D^{2} I(t)+\frac{R}{L} D I(t)+\frac{1}{L C} I(t)=\frac{1}{L} \frac{d v(t)}{d t} .
$$

Let $\omega=\sqrt{1 / L C}$ and $R / L=2 b$. Denote $(1 / L)(d v(t) / d t)$ by $e(t)$. Then, (21) becomes the form

$$
D^{2} I(t)+2 b D I(t)+\omega^{2} I(t)=e(t) .
$$

Generalizing (22) to the fractional order $\beta$ yields

$$
\left(D^{2} I(t)+2 b D I(t)+\omega^{2} I(t)\right)^{\beta}=e(t) .
$$

Below, we specifically study the circuit in Figure 2 with $R=0$, as indicated in Figure 3 .

In the case of Figure 3, (23) becomes the form

$$
\left(D^{2} I(t)+\omega^{2} I(t)\right)^{\beta}=e(t) .
$$

Denote by $h(t)$ the impulse response function of (24). Then, using the techniques in fractional calculus and differential equations [64-81], we have (see [61] for details)

$$
\begin{array}{r}
h(t)=\frac{\sqrt{\pi}}{\Gamma(\beta)(2 \omega)^{\beta-1 / 2}} t^{\beta-1 / 2} J_{\beta-1 / 2}(\omega t), \\
\beta>0, t \geq 0,
\end{array}
$$

where $J_{\beta-1 / 2}(\omega t)$ is the Bessel function of the first kind of $\operatorname{order} \beta-1 / 2$.

The following theorems reflect the particularity of roots of $B^{\beta}(\alpha)$.

Theorem 5. If $0<\beta<1$, all roots of $B^{\beta}(\alpha)$ are located in the left side of the complex plane. 


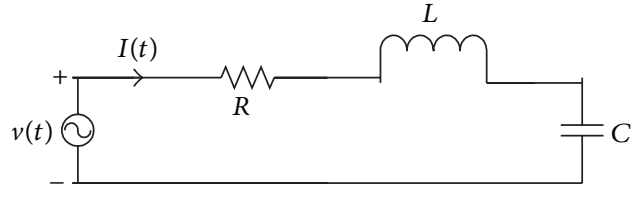

FIGURE 2: Illustration of RLC resonance circuit in series.

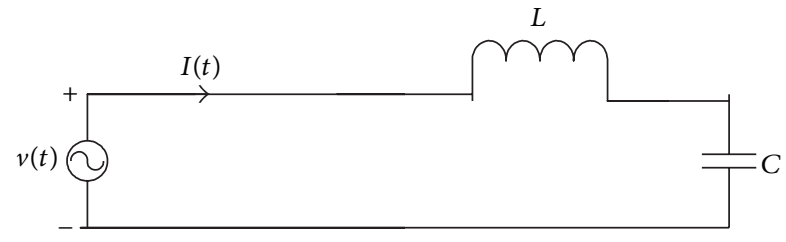

FIGURE 3: Illustration of LC resonance circuit in series with damping $R=0$.

Proof. Note that

$$
J_{v}(t)=\sum_{m=0}^{\infty} \frac{(-1)^{m}}{m ! \Gamma(m+v+1)}\left(\frac{t}{2}\right)^{2 m+v}
$$

From the above, we have the asymptotic expression in the form

$$
J_{v}(t) \sim \frac{1}{\sqrt{t}} \text { for } t \longrightarrow \infty .
$$

Applying (27) to (25) produces

$$
\begin{aligned}
\lim _{t \rightarrow \infty} h(t) & =\lim _{t \rightarrow \infty} \frac{\sqrt{\pi}}{\Gamma(\beta)(2 \omega)^{\beta-1 / 2}} t^{\beta-1 / 2} J_{\beta-1 / 2}(\omega t) \\
& =0, \quad 0<\beta<1 .
\end{aligned}
$$

Denote by $H(s)$ the Laplace transform of $h(t)$. Then, according to the final-value theorem, we have

$$
\lim _{s \rightarrow 0} s H(s)=0, \quad 0<\beta<1 .
$$

The above implies that all poles of $H(s)$ except the origin are strictly in the left side of $s$ plane. In the right of the $s$ plane, $H(s)$ is analytic. This completes the proof.

Theorem 6. If $\beta>1$, at least, parts of roots of $B^{\beta}(\alpha)$ are located in the right side of the complex plane.

Proof. Note that

$$
\begin{aligned}
J_{v}(t)= & \frac{(t / 2)^{v}}{\Gamma(v+1 / 2) \Gamma(1 / 2)} \\
& \times \int_{-1}^{1}\left(1-u^{2}\right)^{v-1 / 2} \cos (t u) d u, \quad \operatorname{Re} v>-\frac{1}{2}
\end{aligned}
$$

From the above, we have the following:

$$
\begin{aligned}
& \frac{-(t / 2)^{v}}{\Gamma(v+1 / 2) \Gamma(1 / 2)} \int_{-1}^{1}\left(1-u^{2}\right)^{v-1 / 2} d u \\
& \quad \leq J_{v}(t) \leq \frac{(t / 2)^{v}}{\Gamma(v+1 / 2) \Gamma(1 / 2)} \int_{-1}^{1}\left(1-u^{2}\right)^{v-1 / 2} d u .
\end{aligned}
$$

Since $\beta>1$ implies $v>1 / 2$, we immediately see that both the right side and the left one on the above expression are respectively unbounded when $t \rightarrow \infty$. Thus, for $\beta>1$, the fractional oscillator (24) is nonstable according to the theory of systems (Gabel and Roberts [77], Dorf and Bishop [78]). Consequently, at least, some of poles of $H(s)$ are in the right of the $s$ plane. Therefore, at least, parts of roots of $B^{\beta}(\alpha)$ are located in the right side of the complex plane.

Most of previous discussions take oscillators of fractional order (24) as a specific object. Note that the number of the characteristic roots of differential equation in general in the form of (12) may also be infinitely great. Hence, comes the following theorem in passing.

Theorem 7. Fractional-order differential equation expressed by (12) has infinite characteristic roots if $n>1$ and if there is at least a pair of roots that are simple complex.

Proof. The characteristic equation of (12) may be decomposed in the form of (18) due to $n>1$. Because there is at least a pair of roots that are simple complex, the number of the characteristic roots of (19) is infinitely great. Thus, the number of characteristic roots of (12) is infinitely great. This completes the proof.

The previous discussions exhibit interesting phenomena of the characteristic roots of the oscillators of the fractional type of (24). In the future, we will work on exploring the answers of the questions described below.

(i) Are all poles of $H(s)$ with respect to (24) in the right of the $s$ plane when $\beta>1$ ?

(ii) Might there be interesting oscillation behavior of (12) if all $c_{j}=0$ in (18) and if $n$ is even?

\section{Conclusions}

We have explained that the number of the characteristic roots of fractional-order oscillators of (24) is usually infinitely great. This conclusion has been further inferred to the case of fractional-order differential equation of (12). We have exhibited that all characteristic roots of (24) are strictly located in the left side of the complex plane if $0<\beta<1$ and at least some of characteristic roots of (24) are in the right side of the complex plane if $\beta>1$. In the case of $\beta=1,(24)$ reduce to an ordinary damping-free oscillator. 


\section{Acknowledgments}

This work was partly supported by the National Natural Science Foundation of China (NSFC) under the project Grant nos. 61272402, 61070214, 60873264, and the 973 plan under the project no. $2011 \mathrm{CB} 302800$.

\section{References}

[1] L. Zhao, Y. Guo, B. Liu, H. Ding, and Q. Li, "A phase-locked oscillator in the high energy physics experiments," in Proceedings of the 7th National Conferenc on Nuclear Electronics and Nuclear Detection Technology, pp. 99-103, Jiangxi, China, October 1994, (Chinese).

[2] Q.-S. Ma, Q.-X. Liu, C. Su, Z.-K. Fan, A.-B. Chang, and H.$\mathrm{Y}$. $\mathrm{Hu}$, "Experimental investigation of the X-band transit-time tube oscillator," High Energy Physics and Nuclear Physics, vol. 27, no. 6, pp. 542-545, 2003 (Chinese).

[3] J.-F. Zang, Q.-X. Liu, Y.-C. Lin, and J. Zhu, "High frequency characteristics of radial three-cavity transmit time oscillator," High Power Laser and Particle Beams, vol. 20, no. 12, pp. 20462050, 2008 (Chinese).

[4] H. Ding et al., "Beijing Spectrometer," High Energy Physics and Nuclear Physics, vol. 16, no. 9, pp. 769-789, 1992 (Chinese).

[5] B. M. Marder, M. C. Clark, L. D. Bacon, J. M. Hoffman, R. W. Lemke, and P. D. Coleman, "The split-cavity oscillator: a high-power E-beam modulator and microwave source," IEEE Transactions on Plasma Science, vol. 20, no. 3, pp. 312-331, 1992.

[6] J. J. Barroso, "Split-cavity monotrons achieving 40 percent electronic efficiency," IEEE Transactions on Plasma Science, vol. 32, no. 3, pp. 1205-1211, 2004.

[7] R. B. Miller, W. F. McCullough, K. T. Lancaster, and C. A. Muehlenweg, "Super-reltron theory and experiments," IEEE Transactions on Plasma Science, vol. 20, no. 3, pp. 332-343, 1992.

[8] R. W. Lemke, "Dispersion analysis of symmetric transverse magnetic modes in a split cavity oscillator," Journal of Applied Physics, vol. 72, no. 9, pp. 4422-4428, 1992.

[9] N. Akhmediev, J. M. Soto-Crespo, and P. Grelu, "Roadmap to ultra-short record high-energy pulses out of laser oscillators," Physics Letters A, vol. 372, no. 17, pp. 3124-3128, 2008.

[10] C. Bachas, "A proof of exponential suppression of high-energy transitions in the anharmonic oscillator," Nuclear Physics B, vol. 377, no. 3, pp. 622-648, 1992.

[11] A. Winter, F. Ö. Ilday, O. D. Mücke et al., "Towards highperformance optical master oscillators for energy recovery linacs," Nuclear Instruments and Methods in Physics Research A, vol. 557, no. 1, pp. 299-304, 2006.

[12] V. V. Dodonov, "Irregular behaviour of a quantum kicked oscillator," Physics Letters A, vol. 214, no. 1-2, pp. 27-32, 1996.

[13] C. Z. Tan, "Electric potential energy of the incident light and the Hamiltonian of the induced oscillators in non-absorbing isotropic dielectrics," Physica B, vol. 269, no. 3-4, pp. 373-378, 1999.

[14] G. A. Diamandis, B. C. Georgalas, A. B. Lahanas, and E. Papantonopoulos, "On the high energy suppression of transition amplitudes in the driven anharmonic oscillator," Physics Letters B, vol. 306, no. 3-4, pp. 319-326, 1993.

[15] Z. Greenwald, D. W. Woodard, A. R. Calawa, and L. F. Eastman, "The effect of a high energy injection on the performance of $\mathrm{mm}$ wave Gunn oscillators," Solid State Electronics, vol. 31, no. 7, pp. 1211-1214, 1988.
[16] P. M. Mathews, M. Seetharaman, S. Raghavan, and V. T. A. Bhargava, "A simple accurate formula for the energy levels of oscillators with a quartic potential," Physics Letters A, vol. 83, no. 3, pp. 118-120, 1981.

[17] D. Faiman, "Harmonic oscillator analysis of the hyperon spectrum," Nuclear Physics B, vol. 32, no. 2, pp. 573-608, 1971.

[18] G. Cocho, J. Flores, and A. Mondragón, “The relativistic harmonic oscillator and nuclear reactions," Nuclear Physics A, vol. 128, no. 1, pp. 110-128, 1969.

[19] M. C. Baldiotti, R. Fresneda, and D. M. Gitman, "Quantization of the damped harmonic oscillator revisited," Physics Letters A, vol. 375, no. 15, pp. 1630-1636, 2011.

[20] H. Kyu Shin, "Probability of vibrational excitations in high energy collisions," Chemical Physics Letters, vol. 3, no. 3, pp. 125127, 1969.

[21] M. W. Kirson, "Oscillator parameters in nuclei," Nuclear Physics A, vol. 781, no. 3-4, pp. 350-362, 2007.

[22] D. M. Clement, "Harmonic oscillator potential insertions in particle lines," Nuclear Physics A, vol. 174, no. 3, pp. 561-571, 1971.

[23] C. M. Sikström, H. Nilsson, U. Litzén, A. Blom, and H. Lundberg, "Uncertainty of oscillator strengths derived from lifetimes and branching fractions," Journal of Quantitative Spectroscopy and Radiative Transfer, vol. 74, no. 3, pp. 355-368, 2002.

[24] M. Asghari, P. Pedram, and K. Nozari, "Harmonic oscillator with minimal lengthminimal momentum, and maximal momentum uncertainties in SUSYQM framework," Physics Letters $B$, vol. 725, no. 4-5, pp. 451-455, 2013.

[25] C.-I. Um, K.-H. Yeon, and T. F. George, "The quantum damped harmonic oscillator," Physics Report, vol. 362, no. 2-3, pp. 63192, 2002.

[26] M. K. Bahar and F. Yasuk, "Exact solutions of the massdependent Klein-Gordon equation with the vector quark-antiquark interaction and harmonic oscillator potential," Advances in High Energy Physics, vol. 2013, Article ID 814985, 6 pages, 2013.

[27] H. Hassanabadi, Z. Molaee, M. Ghominejad, and S. Zarrinkamar, "Spin-one DKP equation in the presence of Coulomb and harmonic oscillator interactions in (1+3)-dimension," Advances in High Energy Physics, vol. 2012, Article ID 489641, 10 pages, 2012.

[28] S. Bhattacharya and S. Roy, "Dissipative effect and tunneling time," Advances in Mathematical Physics, vol. 2011, Article ID 138358, 13 pages, 2011.

[29] N. Saad, R. L. Hall, H. Çiftçi, and Ö. Yeşiltaş, "Study of the generalized quantum isotonic nonlinear oscillator potential," Advances in Mathematical Physics, vol. 2011, Article ID 750168, 20 pages, 2011.

[30] C. Floris, "Stochastic stability of damped Mathieu oscillator parametrically excited by a Gaussian noise," Mathematical Problems in Engineering, vol. 2012, Article ID 375913, 18 pages, 2012.

[31] E. Yaşar, "Integrating factors and first integrals for Liénard type and frequency-damped oscillators," Mathematical Problems in Engineering, vol. 2011, Article ID 916437, 10 pages, 2011.

[32] L. S. Osborne, "A relativistic oscillator model applied to leptonnucleon reactions," Physics Letters B, vol. 63, no. 4, pp. 456-458, 1976.

[33] J. M. Cornwall and G. Tiktopoulos, "Functional Schrödinger equation approach to high-energy multiparticle scattering," Physics Letters B, vol. 282, no. 1-2, pp. 195-200, 1992. 
[34] J. P. Baltanás, J. L. Trueba, and M. A. F. Sanjuán, "Energy dissipation in a nonlinearly damped Duffing oscillator," Physica D, vol. 159, no. 1-2, pp. 22-34, 2001.

[35] A. Erturk and D. J. Inman, "Broadband piezoelectric power generation on high-energy orbits of the bistable Duffing oscillator with electromechanical coupling," Journal of Sound and Vibration, vol. 330, no. 10, pp. 2339-2353, 2011.

[36] K. F. Riley, M. P. Hobson, and S. J. Bence, Mathematical Methods For Physics and Engineering, Cambridge Press, Cambridge, UK, 2006.

[37] T. T. Soong and M. Grigoriu, Random Vibration of Mechanical and Structural Systems, Prentice Hall, New York, NY, USA, 1993.

[38] C. M. Harris, Shock and Vibration Handbook, McGraw-Hill, New York, NY, USA, 4th edition, 1995.

[39] A. Papoulis, Circuits and Systems: A Modern Approach, Oxford University Press, New York, NY, USA, 1995.

[40] J. S. Bendat and A. G. Piersol, Random Data: Analysis and Measurement Procedure, John Wiley \& Sons, New York, NY, USA, 3rd edition, 2000.

[41] S. R. Devasahayam, Signals and Systems in Biomedical Engineering, Springer, New York, NY, USA, 2013.

[42] U. Karrenberg, Signals, Processes, and Systems: An Interactive Multimedia Introduction To Signal Processing, Springer, New York, NY, USA, 2013.

[43] W. A. Edson, "Intermittent behavior in oscillators," Bell System Technical Journal, vol. 24, no. 1, pp. 1-22, 1945.

[44] B. P. Balaban, K. B. Karafin, and S. D. Snyder, "Monte Carlo tolerance analysis of the integrated, single- substrate, RC, Touch-Tone oscillator," Bell System Technical Journal, vol. 50, no. 4, pp. 1263-1291, 1971.

[45] G. A. Korn and T. M. Korn, Mathematical Handbook For Scientists and Engineers, 1.6-3, McGraw-Hill, New York, NY, USA, 1961.

[46] S. G. Krantz, "The fundamental theorem of algebra," in Handbook of Complex Variables, 1.1.7 and 3.1.4, pp. 7 and 32-33, 1999.

[47] Y. E. Ryabov and A. Puzenko, "Damped oscillations in view of the fractional oscillator equation," Physical Review B, vol. 66, no. 18, Article ID 184201, 8 pages, 2002.

[48] W. E. K. Ahmad, R. El-khazali, and A. S. R. Elwakil, "Fractionalorder Wien-bridge oscillator," Electronics Letters, vol. 37, no. 18, pp. 1110-1112, 2001.

[49] A. G. E. Radwan, A. S. Elwakil, and A. M. A. S. Soliman, "Fractional-order sinusoidal oscillators: design procedure and practical examples," IEEE Transactions on Circuits and Systems I, vol. 55, no. 7, pp. 2051-2063, 2008.

[50] A. D. Drozdov, "Fractional oscillator driven by a Gaussian noise," Physica A, vol. 376, no. 1-2, pp. 237-245, 2007.

[51] A. Tofighi and H. N. Pour, " $\varepsilon$-expansion and the fractional oscillator," Physica A, vol. 374, no. 1, pp. 41-45, 2007.

[52] A. Tofighi, "The intrinsic damping of the fractional oscillator," Physica A, vol. 329, no. 1-2, pp. 29-34, 2003.

[53] T. Blaszczyk, M. Ciesielski, M. Klimek, and J. Leszczynski, "Numerical solution of fractional oscillator equation," Applied Mathematics and Computation, vol. 218, no. 6, pp. 2480-2488, 2011.

[54] B. N. Narahari Achar, J. W. Hanneken, and T. Clarke, "Response characteristics of a fractional oscillator," Physica A, vol. 309, no. 3-4, pp. 275-288, 2002.

[55] B. N. Narahari Achar, J. W. Hanneken, and T. Clarke, "Damping characteristics of a fractional oscillator," Physica A, vol. 339, no. 3-4, pp. 311-319, 2004.
[56] S. C. Lim and L. P. Teo, "The fractional oscillator process with two indices," Journal of Physics A, vol. 42, no. 6, Article ID 065208, 2009.

[57] S. C. Lim, L. Ming, and L. P. Teo, "Locally self-similar fractional oscillator processes," Fluctuation and Noise Letters, vol. 7, no. 2, pp. L169-L179, 2007.

[58] S. C. Lim, M. Li, and L. P. Teo, "Langevin equation with two fractional orders," Physics Letters A, vol. 372, no. 42, pp. 63096320, 2008.

[59] S. V. Muniandy and S. C. Lim, "Modeling of locally self-similar processes using multifractional Brownian motion of RiemannLiouville type," Physical Review E, vol. 63, no. 4, Article ID 046104, 7 pages, 2001.

[60] C. H. Eab and S. C. Lim, "Path integral representation of fractional harmonic oscillator," Physica A, vol. 371, no. 2, pp. 303-316, 2006.

[61] M. Li, S. C. Lim, and S. Chen, "Exact solution of impulse response to a class of fractional oscillators and its stability," Mathematical Problems in Engineering, vol. 2011, Article ID 657839, 9 pages, 2011.

[62] J. R. Yu, Complex Functions, High Education Press, 1979, (Chinese).

[63] S. K. Mitra and J. F. Kaiser, Handbook For Digital Signal Processing, John Wiley \& Sons, New York, NY, USA, 1993.

[64] J. Klafter, S. C. Lim, and R. Metzler, Fractional Dynamics: Recent Advances, World Scientific, Singapore, 2012.

[65] R. Hilfer, Applications of Fractional Calculus in Physics, World Scientific, Singapore, 2000.

[66] C. Cattani, "Fractional calculus and Shannon wavelet," Mathematical Problems in Engineering, vol. 2012, Article ID 502812, 26 pages, 2012.

[67] M. H. Heydari, M. R. Hooshmandasl, F. Mohammadi, and C. Cattani, "Wavelets method for solving systems of nonlinear singular fractional Volterra integro-differential equations," Communications in Nonlinear Science and Numerical Simulation, vol. 19, no. 1, pp. 37-48, 2014.

[68] Y. M. Wang, "Maximum-norm error estimates of ADI methods for a two-dimensional fractional sub-diffusion equation," Advances in Mathematical Physics, vol. 2013, Article ID 293706, 10 pages, 2013.

[69] J. S. Duan, "The periodic solution of fractional oscillation equation with periodic input," Advances in Mathematical Physics, vol. 2013, Article ID 869484, 6 pages, 2013.

[70] M. Carlini, T. Honorati, and S. Castellucci, "Photovoltaic greenhouses: comparison of optical and thermal behaviour for energy savings," Mathematical Problems in Engineering, vol. 2012, Article ID 743764, 10 pages, 2012.

[71] M. Carlini and S. Castellucci, "Modelling the vertical heat exchanger in thermal basin," in Computational Science and Its Applications, vol. 6785 of Lecture Notes in Computer Science, pp. 277-286, 2011.

[72] C. Toma, "Advanced signal processing and command synthesis for memory-limited complex systems," Mathematical Problems in Engineering, vol. 2012, Article ID 927821, 13 pages, 2012.

[73] E. G. Bakhoum and C. Toma, "Specific mathematical aspects of dynamics generated by coherence functions," Mathematical Problems in Engineering, vol. 2011, Article ID 436198, 10 pages, 2011.

[74] E. G. Bakhoum and C. Toma, "Transient aspects of wave propagation connected with spatial coherence," Mathematical Problems in Engineering, vol. 2013, Article ID 691257, 5 pages, 2013. 
[75] C. A. Monje, Y. Q. Chen, B. M. Vinagre, D. Xue, and V. Feliu, Fractional Order Systems and Controls-Fundamentals and Applications, Springer, London, UK, 2010.

[76] Y. Q. Chen, B. M. Vinagre, and I. Podlubny, "Fractional order disturbance observer for robust vibration suppression," Nonlinear Dynamics, vol. 38, no. 1-4, pp. 355-367, 2004.

[77] R. A. Gabel and R. A. Roberts, Signals and Linear Systems, John Wiley \& Sons, New York, NY, USA, 1973.

[78] R. C. Dorf and R. H. Bishop, Modern Control Systems, Prentice Hall, New York, NY, USA, 9th edition, 2002.

[79] O. M. Abuzeid, A. N. Al-Rabadi, and H. S. Alkhaldi, "Recent advancements in fractal geometric-based nonlinear time series solutions to the micro-quasistatic thermoviscoelastic creep for rough surfaces in contact," Mathematical Problems in Engineering, vol. 2011, Article ID 691270, 29 pages, 2011.

[80] O. M. Abuzeid, A. N. Al-Rabadi, and H. S. Alkhaldi, "Fractal geometry-based hypergeometric time series solution to the hereditary thermal creep model for the contact of rough surfaces using the Kelvin-Voigt medium," Mathematical Problems in Engineering, vol. 2010, Article ID 652306, 22 pages, 2010.

[81] S. X. Hu, Z. W. Liao, and W. F. Chen, "Sinogram restoration for low-dosed x-ray computed tomography using fractional-order perona-malik diffusion," Mathematical Problems in Engineering, vol. 2012, Article ID 391050, 13 pages, 2012. 

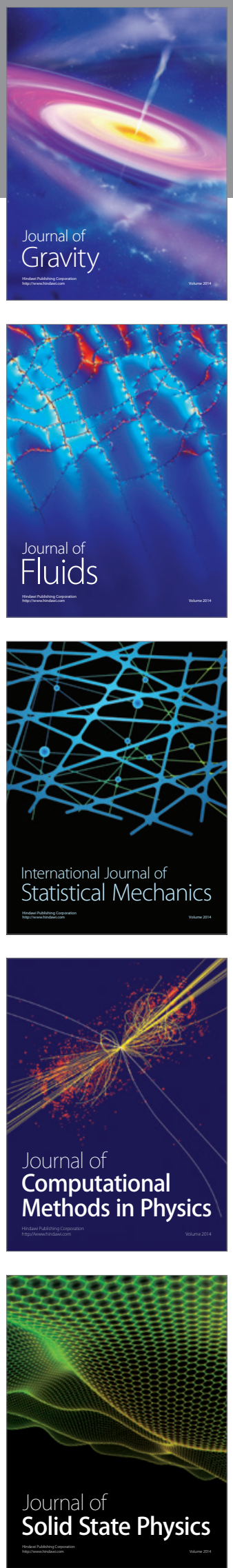

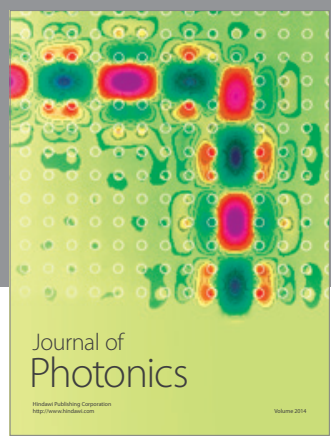

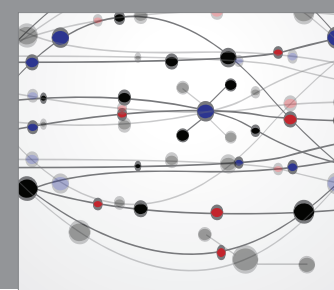

The Scientific World Journal

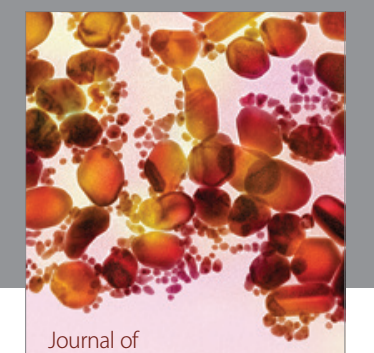

Soft Matter
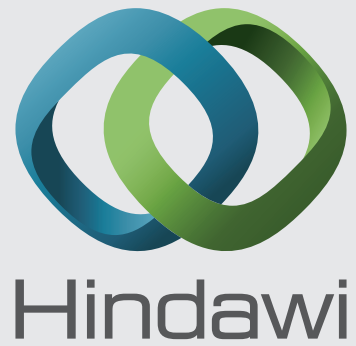

Submit your manuscripts at

http://www.hindawi.com
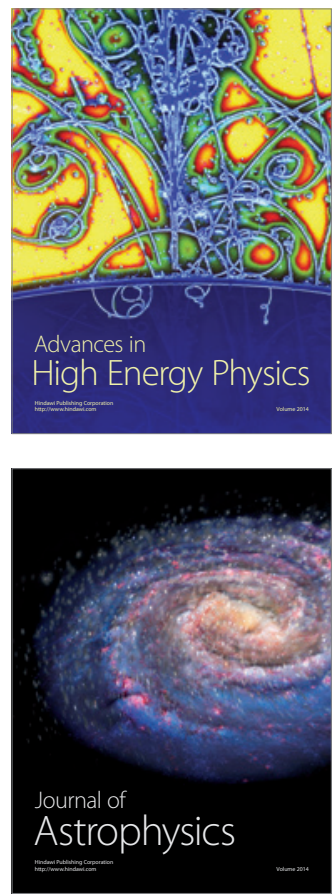
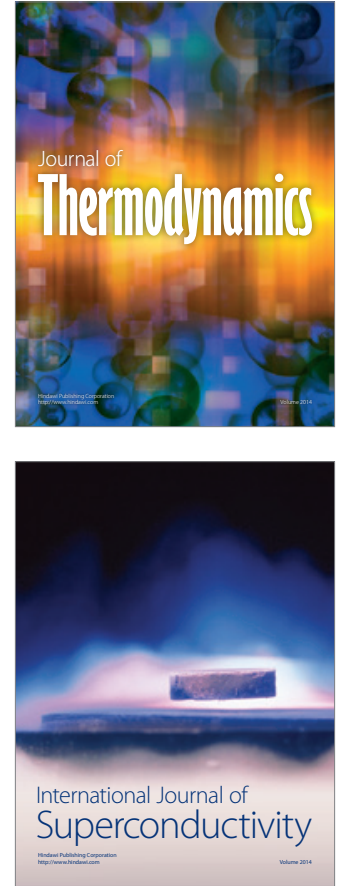
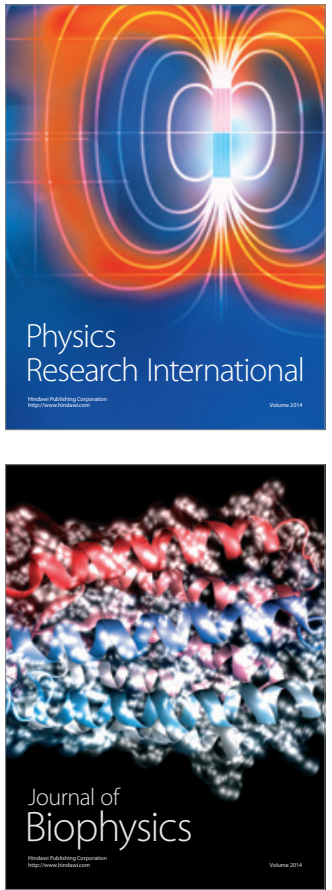
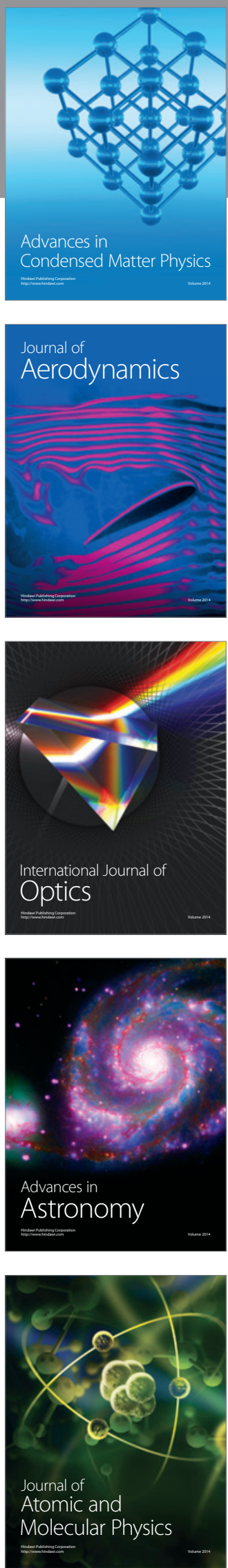